\title{
Generation of Highly-chirped Dissipative Solitons in Er-doped All-fiber Oscillator
}

\author{
I. S. Zhdanov ${ }^{1,2}$, D. S. Kharenko ${ }^{1,2}$, E. V. Podivilov ${ }^{1,2}$, S. A. Babin ${ }^{1,2}$, A. Apolonski ${ }^{2,4}$, \\ A. E. Bednyakova ${ }^{1,3}$, M. P. Fedoruk ${ }^{1,3}$, and S. K. Turitsyn ${ }^{1,5}$ \\ ${ }^{1}$ Novosibirsk State University, Russia \\ ${ }^{2}$ Institute of Automation and Electrometry, Russia \\ ${ }^{3}$ Institute of Computational Technologies, Russia \\ ${ }^{4}$ Ludwig-Maximilians-Universitaet Muenchen and Max-Planck-Institut fuer Quantenoptik, Germany \\ ${ }^{5}$ Aston Institute of Photonic Technologies, Aston University, UK
}

\begin{abstract}
The all-fiber highly-chirped dissipative soliton (HCDS) oscillator was realised at $1.5 \mu \mathrm{m}$ wavelength. A normal net cavity dispersion was achieved by using a conventional dispersion compensating fiber (DCF). To separate effects of the amplitude self-modulation and dissipative soliton formation, we exploit in the laser cavity both standard single mode fiber and polarization maintaining single mode fiber. The properties of the generated pulses have been varied by changing spectral filter bandwidth and DCF lengths. After compression of the nJ-level $\sim 6$ ps HCDS in the external fiber compressor, we measured the output pulse duration of $165 \mathrm{fs}$ (an estimated chirp parameter 40).
\end{abstract}

\section{INTRODUCTION}

All-fiber oscillators are useful tools for nonlinear spectroscopy [1], few-cycle pulse synthesis [2], frequency metrology [3], terahertz-wave generation [4], and optical communications [5]. High power mode-locked fiber lasers generating signal in $\sim 1.5$ micron range have currently attracted a great deal of attention. The recent progress in demonstration of the all-normal dispersion (ANDi) highlychirped dissipative solitons (HCDS) generators in $1 \mu \mathrm{m}$ spectral region $[6, ?]$ stimulated research on transfer of this approach to other spectral intervals.

For the most of practical applications long-term stability and reliability of lasers are equally important as short duration and high power of the generated pulses. From this view point, the allfiber lasers is an attractive solution due to their compact size, no need for complicated adjustments, high efficiency and good beam quality. There is, however, one important drawback - a polarization state that is undefined in standard single-mode fibers (SMF) and that can change uncontrollably because of the the environmental fluctuations, especially in a long fiber cavity. This can be mitigated by the use of the polarization maintaining (PM) fibers. Recent studies have shown that $150 \mathrm{fs}$ pulses can be generated in all-fiber all-PM laser scheme with graphene saturable absorber (GSA) [8]. This laser operates in anomalous net dispersion and energy of pulses does not exceed $25 \mathrm{pJ}$ for the shortest pulse duration and $174 \mathrm{pJ}$ for the longest ( $300 \mathrm{fs}$ ). A slightly higher energy ( $44 \mathrm{pJ}$ ) for $174 \mathrm{fs}$ pulse duration was obtained previously in non-PM cavity with GSA [9]. Topological insulator has shown a great potential as a saturable adsorber (SA) for all-fiber lasers. The shortest pulse duration ( $<130 \mathrm{fs}$ ) for this type of SA in $1.5 \mu \mathrm{m}$ region was demonstrated in [10]. The energy of pulses reached $45 \mathrm{pJ}$ and was significantly increased up to $0.21 \mathrm{~nJ}$ with preserving short pulse duration ( $<170 \mathrm{fs}$ ) in the following work [11]. One should also mention another work [12], in which the authors used all-fiber cavity with nonlinear polarization evolution (NPE) mode-locking to provide $111 \mathrm{fs}$ pulses with the same energy level $(\sim 0.2 \mathrm{~nJ})$. To the best of our knowledge, these are the shortest powerful pulses that have been obtained in all-fiber scheme and characterized carefully with appropriate interferometric auto-correlation technique and/or radio-frequency (RF) spectrum measurements.

It is also well known that a semiconductor saturable absorber mirror (SESAM) gives many advantages for the pulse energy scalability and introduces only a minimal number of bulk optics elements into a fiber laser cavity $[13, ?, ?]$. However, the generated pulses have quite long duration. The shortest one have been demonstrated in [13] (528 fs with $1.8 \mathrm{~nJ}$ energy). The energy of the observed pulses reached $38 \mathrm{~nJ}$, however, their duration also increased up to $700 \mathrm{fs}$ [15]. Finally, only the schemes with NPE mode-locking realized with bulk optics elements can provide femtosecond pulses with high energy and small duration simultaneously $[16, ?, ?]$. Note, that $1480 \mathrm{~nm}$-pump is likely to give some additional benefits in comparison with $980 \mathrm{~nm}$-pump. For instance, the energy 
of pulses generated with $980 \mathrm{~nm}$-pump is three times lower [17] than that obtained in a similar scheme with $1480 \mathrm{~nm}$-pump (3.5 nJ against $10 \mathrm{~nJ}$ ) [18].

In this work we exploit the approach described in [19] for the Yb-doped fiber oscillator and demonstrate a hybrid all-fiber Er-doped active fiber oscillator generating highly-chirped dissipative solitons. Dependence of the generated pulses characteristics on spectral filter bandwidth and DCF length are investigated.

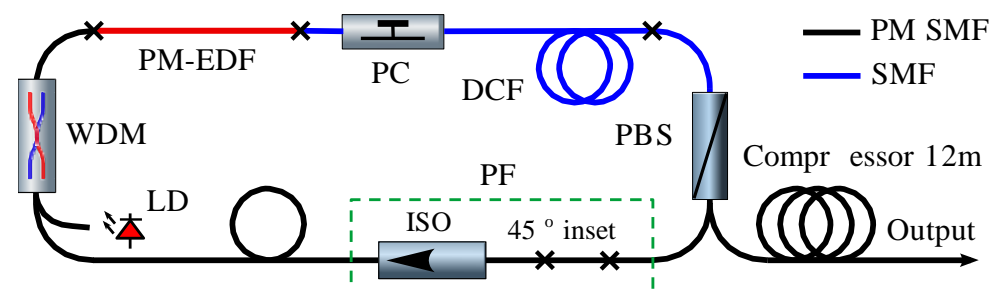

Figure 1. The scheme of an all-fiber oscillator: WDM - wavelength division multiplexer, EDF - Erbiumdoped fiber, PC - polarization controller, DCF - dispersion compensated fiber, PBS - polarization beam splitter, ISO - optical fiber isolator, PF - polarization filter, SMF - single mode fiber, PM SMF - polarization maintaining single mode fiber, LD - laser diode.

\section{EXPERIMENTAL SETUP}

To realize the HCDS special conditions like normal cavity dispersion, sufficient level of amplification and spectral filtering have to be achieved. To provide normal net cavity dispersion at $1550 \mathrm{~nm}$ we used in the scheme dispersion compensated fiber (DCF) with high normal dispersion in this wavelength range.

The laser cavity (see Fig. 1) includes two key parts: conventional single mode fiber (DCF, $\beta_{2}=48 \mathrm{ps}^{2} / \mathrm{km}$ and short pigtails of the polarization beam splitter) and polarization maintaining (PM) single mode fiber (5-m long, $\beta_{2}=-23 \mathrm{ps}^{2} / \mathrm{km}$ ) in order to provide conditions for separate amplitude self-modulation (via nonlinear polarization evolution, NPE) and dissipative soliton formation (driven by nonlinearity and dispersion), accordingly. A Lyot-type fiber polarization filter (PF) determines the spectral range of the circulating dissipative solitons. It contains PM fiber isolator as an analyzer and PM inset spliced with an angle of 45 degree to the main cavity axis as a birefrigent plate. The inset was placed in a temperature controller for changing the spectral position of the transmittance maximum. The laser diode (LD) with maximum output power up to $500 \mathrm{~mW}$ at $980 \mathrm{~nm}$ pumps the 1.3-m PM Erbium-doped fiber (EDF) through the wavelength-division multiplexer (WDM). EDF has anomalous dispersion of about $-20 \mathrm{ps}^{2} 9 \mathrm{~km}$ (Nufern, PM-ESF-7/125). Polarization controller serves for tuning of the NPE mode-locking system to provide stable modelocking. The 12-m fiber compressor dechirpes the generating pulses. The generated HCDSs were characterized by optical spectrum analyzer (Yokogawa 6370), radio-frequency spectrum analyzer (Agilent N9010A), power measurement (Thorlabs Inc.) and interferometric autocorrelator (Avesta Ltd. AA-20DD).

\section{RESULTS AND DISCUSSION}

The following parameters of the cavity have been varied in order to determine their impact on the pulse parameters: DCF length (and respectively a net cavity dispersion), length of the spectral inset which determined properties of the filter. The pump power and PC were adjusted to obtain a stable mode-locking at fundamental repetition frequency with the highest energy. A set of the examined regimes in the plane (DCF length, PF bandwidth) is presented by dots on Fig. 2(a). Solid-line boxes combine the spectra into the groups in which a comparison was made. A regime selected for a more detailed investigation is marked with a red triangle.

The NPE mode-locking stability and the value of net cavity dispersion limit the DCF length variation range from above and below accordingly. The DCF span does not preserve the polarization state, therefore, its lengthening could result in NPE-overdriving. Thus 13-m, 9-m and 6-m long DCF were used in the experiment. Comparison of the output spectra with different DCF lengths and PF bandwidths is depicted in Figs. 2(b)-(d). We have found that the net cavity dispersion decrease (by shortening DCF length) resulted in a wider spectra of the generated HCDS. In these cases (Fig. 2(b) and Fig. 2(c)) the cavities have the same PF bandwidth, so the pulse spectral 


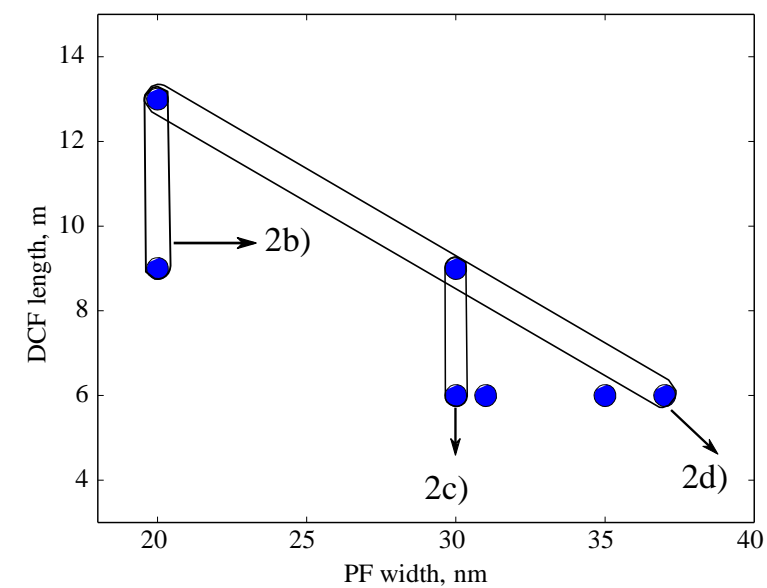

(a)

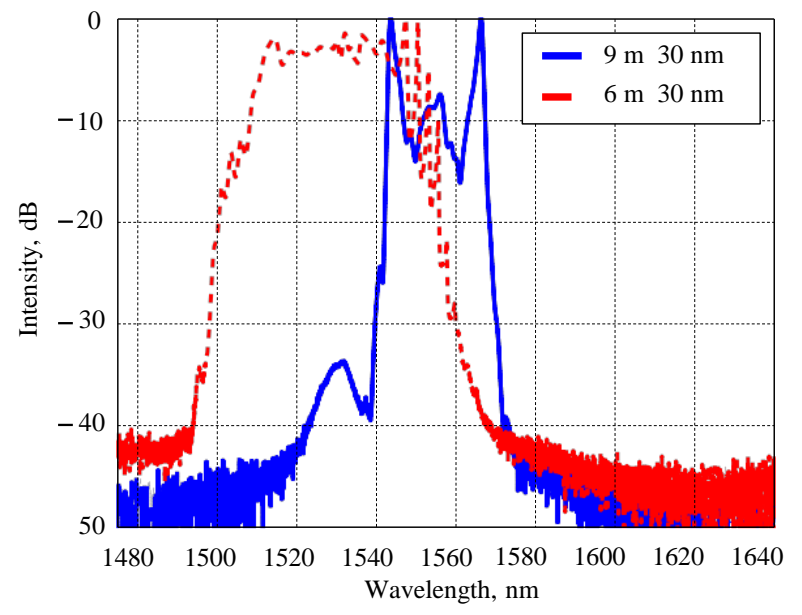

(c)

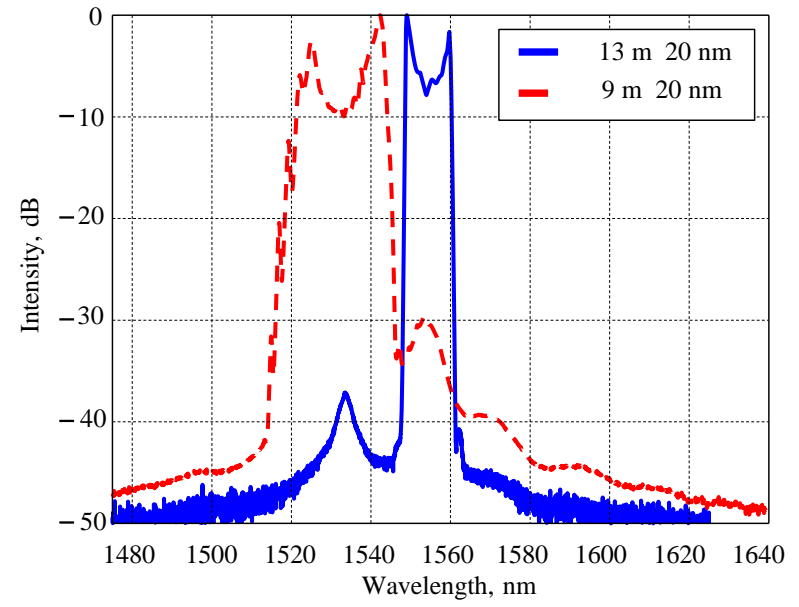

(b)

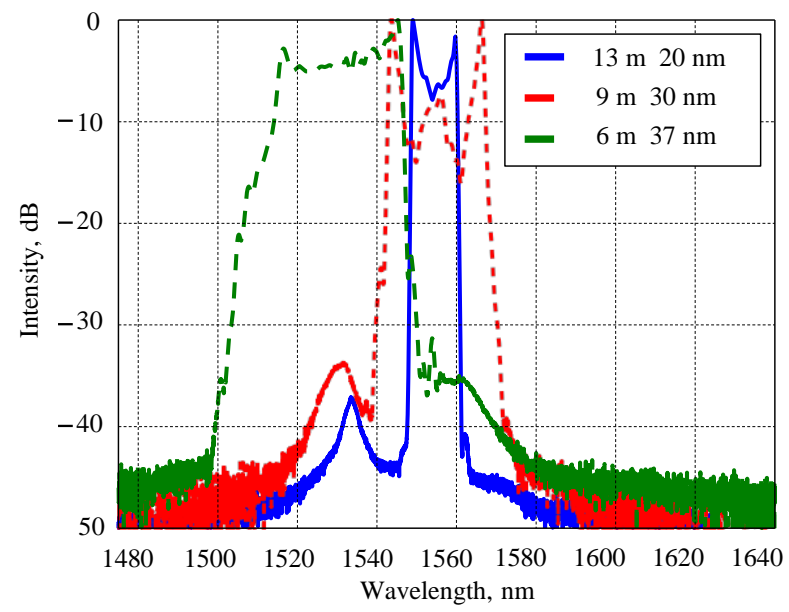

(d)

Figure 2. Comparison of the output pulse spectrum: (a) The set of configurations represented at DCF length and PF width plane; Comparisons: (b) 13-m and 9-m DCF configurations with 20-nm wide filter; (c) 9-m and 6-m DCF configurations with 30-nm wide filter; (d) 13-m, 9-m and 6-m with maximum suitable spectral filter.

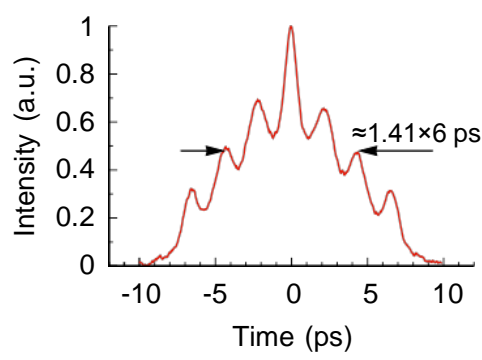

(a)

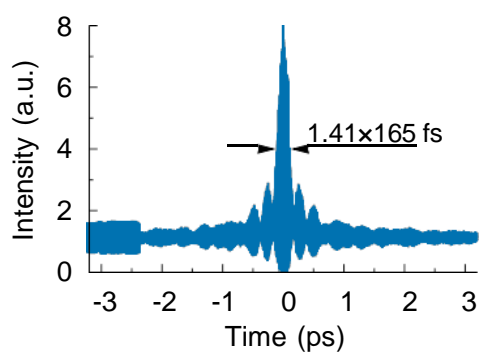

(b)

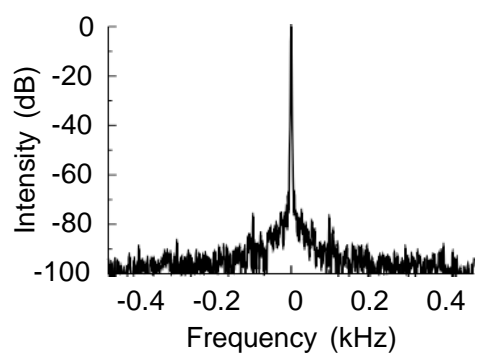

(c)

Figure 3. (a) Intensity ACF of the chirped pulse. (b) Interferometric ACF of the compressed pulse. (c) Typical radio-frequency beat signal centered at fundamental repetition rate (17.3 MHz for 6-m long DCF).

bandwidth becomes wider than the PF bandwidth and cuts by it (Fig. 2(d)). Note that it is preferable to generate the HCDS with a wider spectra as it can be compressed into shorter pulse. Thus, the DCF length and PF bandwidth have been changed simultaneously (Fig. 2(d)). Here the spectrum width increases gradually without any degradation. To produce a stable operation regime in the 13-m long DCF configuration the 20-nm filter was used, but its width was increased up to 37-nm for the 6-m long DCF configuration, where the HCDS with the most broad spectra was obtained. Also the NPE mode-locking was more stable in this configurations. On the other hand, it was shown that a stronger spectral filtering could lead to higher pulse energy [7]. We 


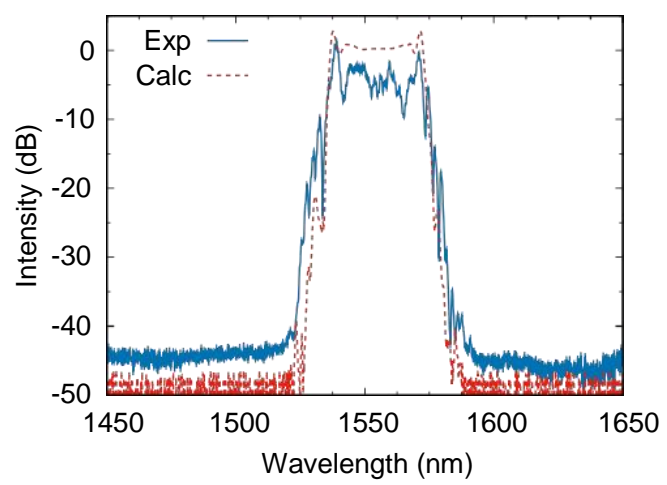

Figure 4. Comparison of the experimental spectrum and the results of numerical modelling for selected configuration.

used, therefore, the configuration with 6-m long DCF part and 28-nm width spectral filter (pointed with a red triangle in Fig. 2(a)). In this scheme we were able to significantly increase the pump power in order to generate pulses with higher energy and wider optical spectra. Corresponding net cavity dispersion was about $0.17 \mathrm{ps}^{2}$. The duration of the pulses was respectively $\sim 6 \mathrm{ps}$ and $165 \mathrm{fs}$ for chirped and de-chirped pulses (Figs. 3(a), (b)). The pulse energy reached $\sim 1 \mathrm{~nJ}$ that was the highest obtained value in the experiment. The pulse train stability was characterized by $\mathrm{RF}$ beat signal measurements (see Fig. 3(c) which were performed at fundamental frequency with $1 \mathrm{~Hz}$ bandwidth in $1 \mathrm{kHz}$ range. RF spectrum demonstrates a high contrast level exceeding $90 \mathrm{~dB}$. Fig. 4 demonstrates that the generated spectra are in a good agreement with the theoretical analysis which was performed by using the generalized nonlinear Schrödinger equation. This model was developed previously and described in detail in [20]. The simulation results show that the laser can be treated as a passive self-similar oscillator, in which pulse shaping is based on both the spectral filtering of chirped pulse and its self-similar evolution in DCF.

\section{CONCLUSION}

In this work we have shown the possibility to separate effects of the amplitude self-modulation and dissipative soliton formation at $1.5 \mu \mathrm{m}$ wavelength range by using PM and non-PM fibers in all-fiber cavity. Stable regimes corresponding to different DCF length, filter parameters and position of the spectral maximum were observed. We found that the most stable powerful HCDSs with the widest optical spectrum can be generated at the net cavity dispersion of $0.17 \mathrm{ps}^{2}$. The spectral width of $\mathrm{PF}$ in the described configuration was $28 \mathrm{~nm}$. After compression of the $\sim 6 \mathrm{ps}$ HCDSs in the $12 \mathrm{~m}$ long external fiber compressor, the final pulse duration was equal to $165 \mathrm{fs}$ (chirp parameter 40).

\section{FUNDING INFORMATION}

This work is supported by Russian Foundation of Basic Research (RFBR) and SB RAS integration project.

\section{REFERENCES}

1. Marangoni, M., A. Gambetta, C. Manzoni, et al., "Fiber-format CARS spectroscopy by spectral compression of femtosecond pulses from a single laser oscillator," Opt. Lett., Vol. 34, No. 21, 3262-3264, 2009.

2. Krauss, G., S. Lohss, T. Hanke, et al., "Synthesis of a single cycle of light with compact erbium-doped fibre technology," Nat. Photonics, Vol. 4, No. 1, 33-36, 2009.

3. Newbury, N. R. and W. C. Swann, "Low-noise fiber-laser frequency combs (Invited)," J. Opt. Soc. Am. B, Vol. 24, No. 8, 1756-1770, 2007.

4. Schneider, A., M. Stillhart, and P. Günter, "High efficiency generation and detection of terahertz pulses using laser pulses at telecommunication wavelengths," Opt. Express, Vol. 14, No. 12, 5376, 2007.

5. Kim, J. and Y. Song, "Ultralow-noise mode-locked fiber lasers and frequency combs: principles, status, and applications," Adv. Opt. Photonics, Vol. 8, No. 3, 465, 2016.

6. Lefrançois, S., K. Kieu, Y. Deng, et al., "Scaling of dissipative soliton fiber lasers to megawatt peak powers by use of large-area photonic crystal fiber," Opt. Lett., Vol. 35, No. 10, 1569-1571, 2010. 
7. Kharenko, D. S., V. A. Gonta, and S. A. Babin, " $50 \mathrm{~nJ} 250 \mathrm{fs}$ all-fibre Raman-free dissipative soliton oscillator," Laser Phys. Lett., Vol. 13, No. 2, 25107, 2016.

8. Tarka, J., J. Boguslawski, G. Sobon, et al., "Power scaling of an all-PM fiber Er-doped modelocked laser based on graphene saturable absorber," IEEE J. Sel. Top. Quantum Electron., Vol. 23, No. 1, 1-6, 2017.

9. Popa, D., Z. Sun, F. Torrisi, et al., "Sub $200 \mathrm{fs}$ pulse generation from a graphene mode-locked fiber laser," Appl. Phys. Lett., Vol. 97, No. 20, 16-19, 2010.

10. Sotor, J., G. Sobon, and K. M. Abramski, "Sub-130 fs mode-locked Er-doped fiber laser based on topological insulator," Opt. Express, Vol. 22, No. 11, 13244, 2014.

11. Boguslawski, J., G. Sobon, R. Zybala, and J. Sotor, "Dissipative soliton generation in Er-doped fiber laser mode-locked by Sb_2Te_3 topological insulator," Opt. Lett., Vol. 40, No. 12, 2786, 2015.

12. Nikodem, M. and K. M. Abramski, "169 MHz repetition frequency all-fiber passively modelocked erbium doped fiber laser," Opt. Commun., Vol. 283, No. 1, 109-112, 2010.

13. Cabasse, A., G. Martel, and J. L. Oudar, "High power dissipative soliton in an Erbium-doped fiber laser mode-locked with a high modulation depth saturable absorber mirror," Opt. Express, Vol. 17, No. 12, 9537-9542, 2009.

14. Cabasse, A., D. Gaponov, K. Ndao, et al., " $130 \mathrm{~mW}$ average power, $4.6 \mathrm{~nJ}$ pulse energy, $10.2 \mathrm{ps}$ pulse duration from an $\mathrm{Er}^{3+}$ fiber oscillator passively mode locked by a resonant saturable absorber mirror," Opt. Lett., Vol. 36, No. 14, 2620, 2011.

15. Tang, M., H. Wang, R. Becheker, etal., "High-energy dissipative solitons generation from a large normal dispersion Er-fiber laser," Opt. Lett., Vol. 40, No. 7, 1414-1417, 2015.

16. Chichkov, N. B., K. Hausmann, D. Wandt, et al., " 50 fs pulses from an all-normal dispersion erbium fiber oscillator,” Opt. Lett., Vol. 35, No. 16, 3081-3083, 2010.

17. Liu, H., Zh. Liu, E. S Lamb, and F. W. Wise., "Self-similar erbium-doped fiber laser with large normal dispersion," Opt. Lett., Vol. 39, No. 4, 1019-1021, 2014.

18. Ruehl, A. V. Kuhn, D. Wandt, and D. Kracht., "Normal dispersion erbium-doped fiber laser with pulse energies above $10 \mathrm{~nJ}$," Opt. Express, Vol. 16, No. 5, 3130, 2008.

19. Kharenko, D. S., E. V. Podivilov, A. A. Apolonski, and S. A. Babin, "20 nJ $200 \mathrm{fs}$ all-fiber highly-chirped dissipative soliton oscillator," Opt. Lett., Vol. 37, No. 19, 4104-4106, 2012.

20. Bednyakova, A. E., S. A. Babin, D. S. Kharenko, et al., "Evolution of dissipative solitons in a fiber laser oscillator in the presence of strong Raman scattering," Opt. Express, Vol. 21, No. 18, 20556-20564, 2013. 